\title{
Fertilité des rameaux anticipés de vigne (Vitis vinifera L.) : II. - Influence de la fertilité potentielle et de la vitesse de croissance
}

\author{
Chantal OLIVAIN et Roger BESSIS \\ Université de Bourgogne, Laboratoire des sciences de la vigne, Faculté des sciences Mirande, B.P. 138, F 21004 \\ Dijon Cedex
}

RÉSUMÉ Certains facteurs qui influent sur l'expression de la fertilité des rameaux anticipés obtenus après écimage sont
analysés. La fertilité observée dépend de la fertilité potentielle des bourgeons anticipés au moment de leur entrée
en croissance et des caractères de cette croissance. La fertilité maximale est obtenue au vignoble lorsque la ferti-
lité potentielle vient d'atteindre sa valeur maximale dans les bourgeons anticipés. Les causes d'une perte de ferti-
lité aux écimages précoces et tardifs sont abordées.

Mot clé additionnel : Ecimage.

Lateral shoot fertility of grapevine (Vitis vinifera L.). II. Influence of potential fertility and growth kinetics.

We have studied some factors influencing the expression of the lateral shoot fertility which develops after decapitation of grapevine. The observed fertility depended on the potential fertility of the lateral buds at the time they began growing and on the characteristics of this growth. Maximum fertility in the vineyard was obtained only when potential fertility reached its maximum value in the lateral buds. The reasons for the lower fertility observed after early and late decapitation are also examined.

Additional key word : Decapitation.

\section{INTRODUCTION}

Lors d'un précédent article (OLIVAIN \& BESSIS, 1988) nous avons montré que les conditions d'écimage interviennent sur l'expression de la fertilité des rameaux anticipés. L'objectif de ce travail est d'étudier quelques-uns des facteurs qui agissent à travers l'importance et l'époque d'écimage. Nous avons retenu deux paramètres : la fertilité potentielle des bourgeons ou des petits rameaux anticipés avant écimage et la cinétique de croissance des rameaux anticipés obtenus après écimage du sarment principal.

\section{MATÉRIEL ET MÉTHODES}

Les conditions expérimentales ont été décrites précédemment (OLIVAIN \& BESSIS, 1988), nous avons limité notre étude aux bourgeons anticipés de rang $6: 10$ éci- mages différents ont donc été pratiqués au-dessus du nœud 6 de la mi-mai jusqu'à la fin juin.

A chaque écimage, nous avons réalisé 2 lots expèrimentaux : dans ces 2 lots, tous les anticipés sont maintenus sur le sarment écimé mais seul l'anticipé situé sous la décapitation fait l'objet de mesures.

- Pour l'un des lots, nous prélevons les anticipés le jour de l'écimage, ce lot composé d'une trentaine d'échantillons permet d'établir la fertilité potentielle des anticipés c'est-à-dire le nombre de grappes potentielles présentes dans les anticipés avant chaque écimage. Les mesures de fertilité sont effectuées grâce à l'étude de coupes sériées pratiquées sur les anticipés fixés au F.A.A. * et inclus dans la paraffine (OLIVAIN \& BESSIS, 1987). Nous avons considéré comme grappe

* Composition du F.A.A. utilisé : 1 volume d'aldéhyde formique (solution à $37 \%$ ), 1 volume d'acide acétique cristallisable, 18 volumes d'alcool éthylique à $50^{\circ}$. 
potentielle tout organe oppositifolié engagé dans le processus de différenciation inflorescentielle. Lors des premiers écimages, on s'adresse à des inflorescences avec un bras interne en forme de dôme élargi où l'on devine l'ébauche des premières ramifications secondaires. Au fur et à mesure des écimages, les inflorescences présentent des ramifications tertiaires puis des boutons floraux.

- Le deuxième lot composé d'une quarantaine de pousses sert à évaluer l'expression de cette fertilité potentielle : fertilité exprimée et fertilité pratique (OLIVAIN \& BESSIS, 1988). La cinétique de croissance des anticipés développés après écimage est étudiée, elle est basée sur des mesures de longueur faites tous les 8 à 10 jours depuis la date d'écimage jusqu'à la dernière mesure de fertilité permettant d'estimer la fertilité pratique.

Lors des deux derniers écimages, nous avons cherché à augmenter la vitesse de croissance des anticipés. Dans ce but, nous avons constitué un troisième lot expérimental où seul l'anticipé situé sous la décapitation est gardé sur le sarment écimé. Les mesures de fertilité et de croissance sont menées de façon identique à celles du deuxième lot.

Nous avons également constitué un lot témoin d'une trentaine de pousses permettant de suivre la croissance des anticipés de rang 6 en l'absence d'écimage.

\section{RÉSULTATS}

\section{A. Analyse de la fertilité potentielle des anticipés}

Nous avons analysé durant une période d'un mois et demi la mise en place de la fertilité potentielle (fig. 1).
Depuis le 11 mai jusqu'au 29 juin, on peut distinguer 3 périodes :

- Une première période allant jusque vers le 17 mai où aucun organe oppositifolié n'est engagé dans la différenciation en inflorescence. Au 17 mai, la grande majorité des organes oppositifoliés ne dépassent pas le stade « anlage » défini par ALLEWELDT \& BALKEMA (1965).

- Une deuxième période après le 17 mai et jusqu'aux environs du 9 juin où le premier organe oppositifolié et à un moindre degré le deuxième s'organisent en inflorescences. Vers le 9 juin, la fertilité potentielle maximale est atteinte.

- Une troisième période après le 9 juin où aucune inflorescence supplémentaire ne se forme. La fertilité potentielle reste stationnaire tandis que les inflorescences présentes poursuivent leur différenciation ; pourtant, l'organogenèse inflorescentielle n'est pas continue jusqu'à la fin juin. Au niveau du premier organe oppositifolié, nous avons déjà signalé que l'état de différenciation des inflorescences n'évolue pas entre le 14 et le 29 juin (OLIVAIN \& BESSIS, 1987) ; les diverses inflorescences se trouvent bloquées soit au stade ramifications secondaires $(21,9 \%)$, soit au stade ramifications tertiaires $(34,4 \%)$, soit au stade boutons floraux $(43,7 \%)$.

En définitive, la fertilité potentielle d'un anticipé de rang 6 atteint au mois de juin une valeur moyenne de 1,8 grappe. Cette fertilité se situe principalement sur le premier organe oppositifolié qui possède une inflorescence bien organisée. La fertilité potentielle du deuxième organe n'est alors que de 0,7 grappe et celleci est moins riche en ramifications. Il est rare de déceler une structure inflorescentielle au niveau du troisième organe oppositifolié, soit il n'a pas dépassé le stade « anlage », soit il a une structure de vrille.

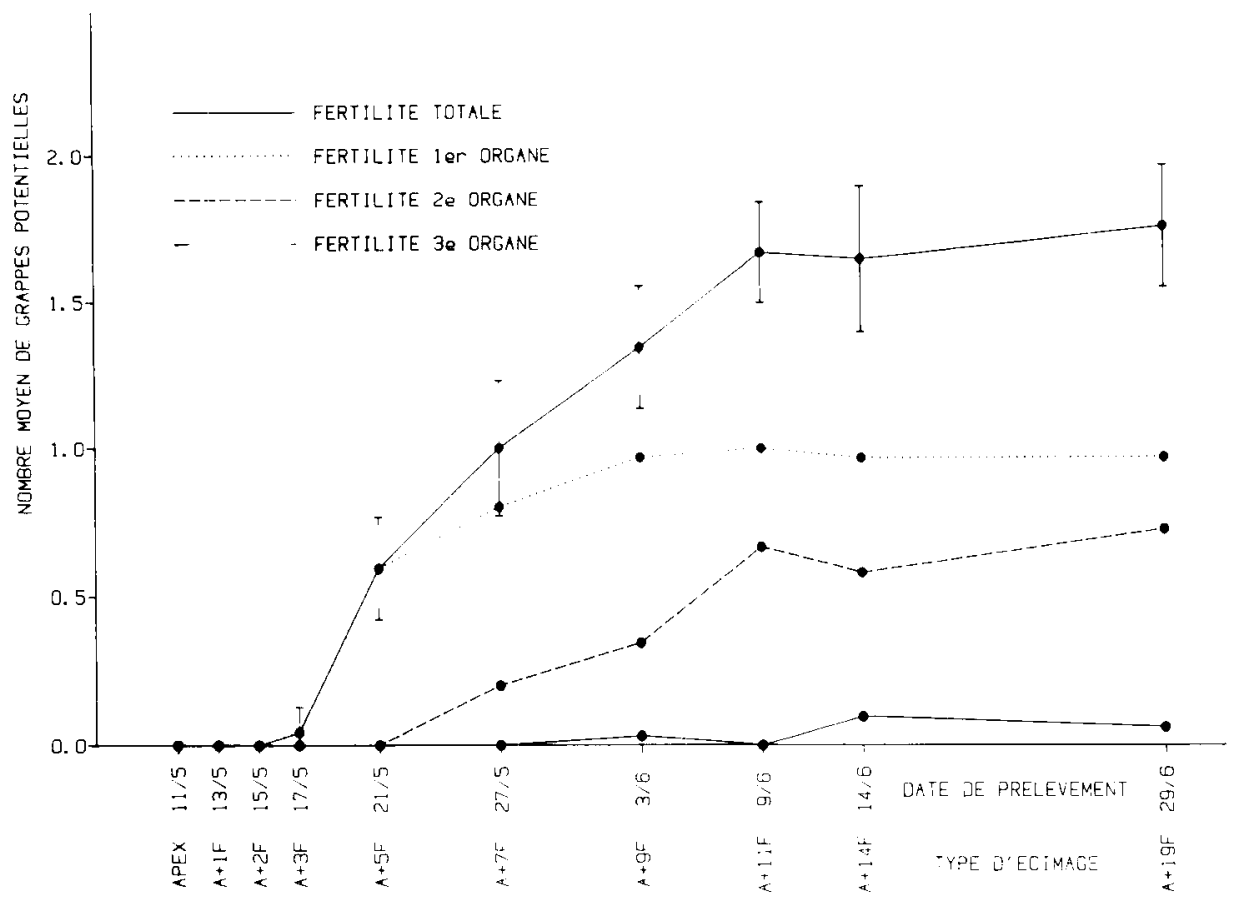

Figure 1

Mise en place de la fertilité potentielle au niveau des anticipés de rang 6 portés par des sarments non écimés (intervalle de confiance pour un coefficient de sécurité de 95 p. 100\%.

Development of the potential fertility in the lateral at position 6 on non-decapitated shoots (confidence limits with a $95 \%$ confidence limit). 


\section{B. Cinétique de croissance des anticipés développés après écimage (fig. 2)}

La croissance des anticipés développés après écimage ou « anticipés forcés » est comparée à celle des anticipés présents sur sarments non écimés ou "anticipés témoins $"$.

Sur sarments non écimés, on note une amorce de la croissance des anticipés, début juin, mais celle-ci reste très faible. De plus, cette croissance est hétérogène, à titre d'exemple, les valeurs extrêmes relevées sont de $0,5 \mathrm{~cm}$ pour la valeur minimale et de $54 \mathrm{~cm}$ pour la valeur maximale. Comme l'a rapporté BouARD (1966), les anticipés insérés à un nœud dépourvu de vrille ou de grappe ont une longueur moyenne supérieure $(10,3 \mathrm{~cm})$ à celle des anticipés insérés à un nœud muni d'une vrille ou d'une grappe $(4,2 \mathrm{~cm})$.

Sur sarments écimés, la croissance des anticipés est importante puisque les rameaux peuvent atteindre plus d'un mètre, elle est également beaucoup plus homogène et le rythme de croissance décrit par BOUARD (1966) ne se maintient pas. Les courbes de croissance montrent une profonde analogie, pourtant, plusieurs particularités les différencient et méritent d'être approfondies :

- Après écimage, il existe une période où la croissance des anticipés forcés n'est pas très différente de celle des anticipés témoins. Cette phase de latence est suivie d'une phase d'accélération mais la phase de latence n'est pas équivalente pour les divers écimages et la croissance des anticipés forcés se différencie plus ou moins rapidement de celle des anticipés témoins. Lors des écimages précoces, la phase de latence est longue pour les écimages du début mai (de l'ordre de $20 \mathrm{j}$ ) puis elle diminue progressivement jusqu'à une valeur de $6 \mathrm{j}$ environ pour l'écimage " apex $+7 \mathrm{~F}$ » réalisé le 27 mai. Pour tous ces traitements l'accélération de la croissance des anticipés a donc lieu presque simultanément, fin mai. Aux écimages suivants, la phase de latence reste faible et sensiblement identique (environ $6 \mathrm{j}$ ), la croissance des anticipés forcés se distingue alors assez rapidement de celle des anticipés témoins mais pour tous ces traitements la phase d'accélération intervient de plus en plus tardivement du fait des dates d'écimage de plus en plus tardives.

- Après la phase d'accélération, il s'établit une période de croissance active où les anticipés atteignent leur vitesse maximale de croissance. Le ralentissement de la croissance se produit en même temps pour tous les anticipés, vers la mi-juillet, comme pour les rameaux principaux. La durée de la période de croissance active est ainsi maximale pour les écimages du mois de mai et elle est progressivement raccourcie pour les écimages du mois de juin. La vitesse maximale de croissance des anticipés est proche de celle du sarment principal (FOURNIOUX \& BESSIS, 1980) ; elle est d'environ $3 \mathrm{~cm}$ par jour excepté pour les 2 derniers écimages. Pour l'écimage " apex $+14 \mathrm{~F}$ 》, la vitesse maximale de croissance est de $2,5 \mathrm{~cm}$ par jour, pour l'écimage « apex + 19 F », elle est de 1,8 cm par jour. Pour ce dernier traitement effectué le 29 juin, le ralentissement général de la croissance a lieu peu de temps après la phase d'accélération due à l'écimage et la vitesse maximale de croissance est notablement plus faible.

Cette analyse fait ressortir les points suivants :

- Les écimages effectués au mois de mai provoquent toujours une entrée en croissance précoce des anticipés malgré un certain temps de latence. Ces anticipés bénéficient d'une période de croissance active assez longue et la vitesse maximale de croissance est voisine de celle du sarment principal. Le temps de latence est assez long pour les écimages du début mai : il peut être relié à la présence de jeunes feuilles inhibitrices sur le sarment écimé, leur nombre diminuant

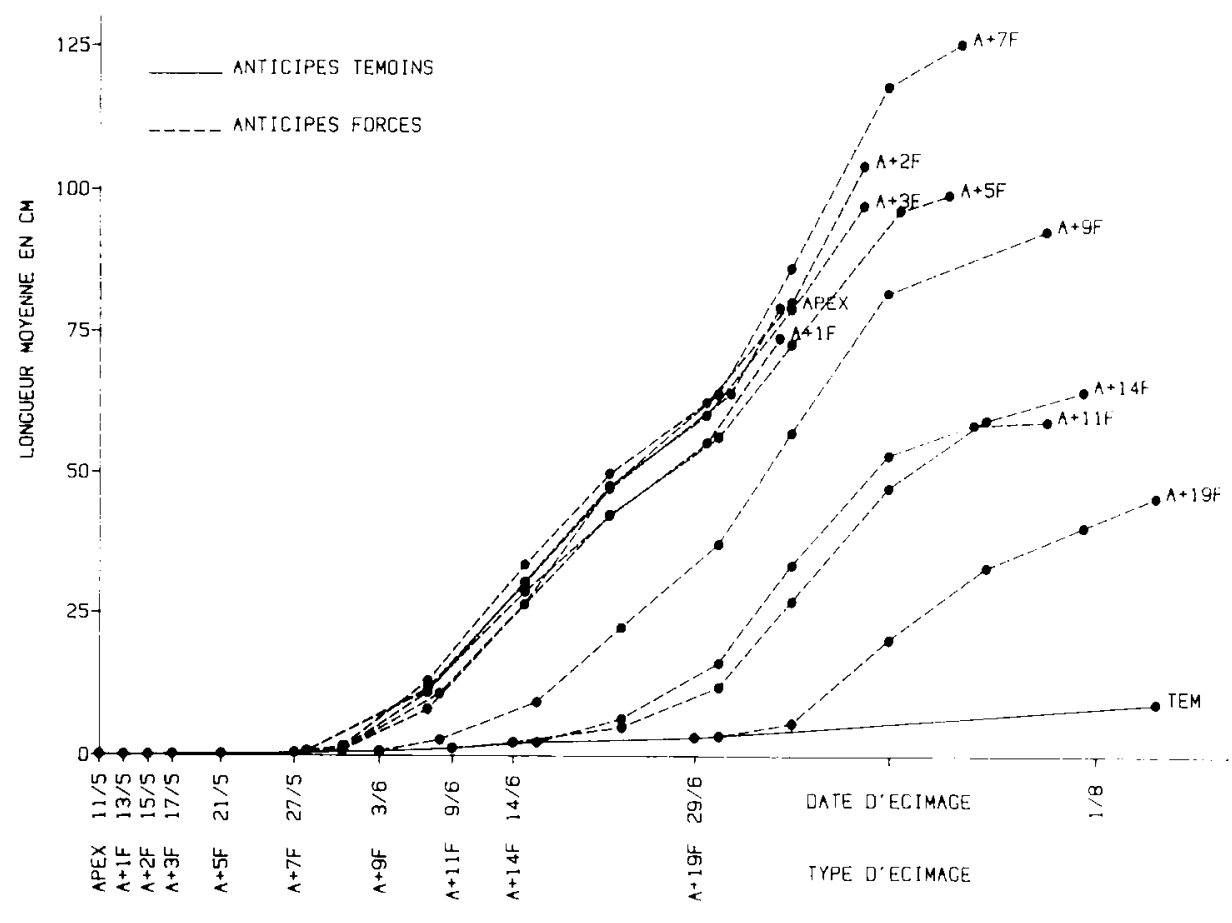

Figure 2

Cinétique de croissance des rameaux anticipés de rang 6 après divers écimages. 
avec l'importance de l'écimage, et à l'état de l'anticipé dont l'organisation augmente avec l'importance de l'écimage. Par ailleurs, en raison des conditions climatiques, la vitesse de croissance de la vigne est, à cette époque, encore relativement lente. Enfin, nous avons constaté que lors de ces écimages, les anticipés inférieurs ont, dans les premiers stades de l'expérimentation, une élongation égale ou supérieure à celle de l'anticipé envisagé : les anticipés inférieurs plus organisés et à l'aisselle de feuilles adultes auraient dans un premier temps la prépondérance.

- Les écimages du début juin permettent une croissance continue des anticipés, en effet, l'accélération de la croissance due à l'écimage coïncide avec l'amorce de croissance des anticipés témoins sur sarments non écimés. Ces anticipés ont une période de croissance active plus courte et la vitesse maximale de croissance reste voisine de celle du sarment principal. Il est intéressant de mentionner que les courbes de croissance des anticipés forcés les 9 et 14 juin après les écimages « apex +11 et $14 \mathrm{~F}$ » sont presque semblables, pourtant, les fertilités pratiques obtenues sont différentes : pour l'écimage « apex + $11 \mathrm{~F}$ » la fertilité pratique est maximale (1,6 grappe par rameau) et pour l'écimage "apex $+14 \mathrm{~F}$ » cette fertilité commence à diminuer (1,1 grappe par rameau) du fait de la chute des grappes.

- L'écimage du 29 juin intervient sur des anticipés dont la croissance a été inhibée pendant un certain temps ; de plus, la vitesse maximale de croissance est fortement diminuée ainsi que la durée de la période de croissance active. Dans un premier temps, nous avons pensé que cette baisse de la vitesse de croissance était la cause de la chute importante des grappes, la fertilité pratique n'étant plus que de 0,3 grappe par rameau.
C. Cinétique de croissance et fertilité des anticipés développés après écimage, dans le cas où seul l'anticipé situé sous la décapitation est maintenu sur le sarment (fig. 3)

Les courbes de croissance sont comparées à celles que nous venons de décrire pour les écimages " apex +14 et $19 \mathrm{~F}$ ». Le début de la croissance (phase de latence et phase d'accélération) n'est pas modifié mais, dans les 2 cas, la vitesse de croissance des anticipés pendant la période de croissance active est accélérée. Pour l'écimage «apex + $14 \mathrm{~F}$ », la vitesse maximale de croissance atteint $4,5 \mathrm{~cm}$ par jour alors que précédemment elle n'était que de 2,5. La vitesse de croissance est donc supérieure à celle observée dans le cas général pour l'écimage " apex $+11 \mathrm{~F}$ 》 $(3 \mathrm{~cm}$ par jour) où nous obtenons la fertilité maximale et un pourcentage de chute des grappes très faible. Pour l'écimage « apex $+19 \mathrm{~F}$ », la vitesse maximale de croissance est de $2,6 \mathrm{~cm}$ par jour contre 1,8 précédemment : elle est du même ordre que la vitesse observée lors de l'écimage « apex $+14 \mathrm{~F}$ » dans le cas général $(2,5 \mathrm{~cm}$ par jour). Rappelons que pour ce traitement le pourcentage de chute des grappes est très inférieur à celui obtenu dans le traitement " apex $+19 \mathrm{~F}$ » où la presque totalité des grappes tombe).

Les valeurs de la fertilité (fertilité exprimée et fertilité pratique) sont reportées au tableau 1 et également comparées à celles obtenues dans le cas général où tous les anticipés sont gardés sur le sarment écimé. Les différentes valeurs sont très proches les unes des autres et la chute des grappes se produit sensiblement dans les mêmes proportions : pour l'écimage « apex $+14 \mathrm{~F}$ ", le pourcentage de chute des grappes est de $26,6 \%$ contre 37,5 dans le cas général, pour l'écimage « apex $+19 \mathrm{~F}$ » il est de $84,6 \%$ contre 81,2 dans le cas

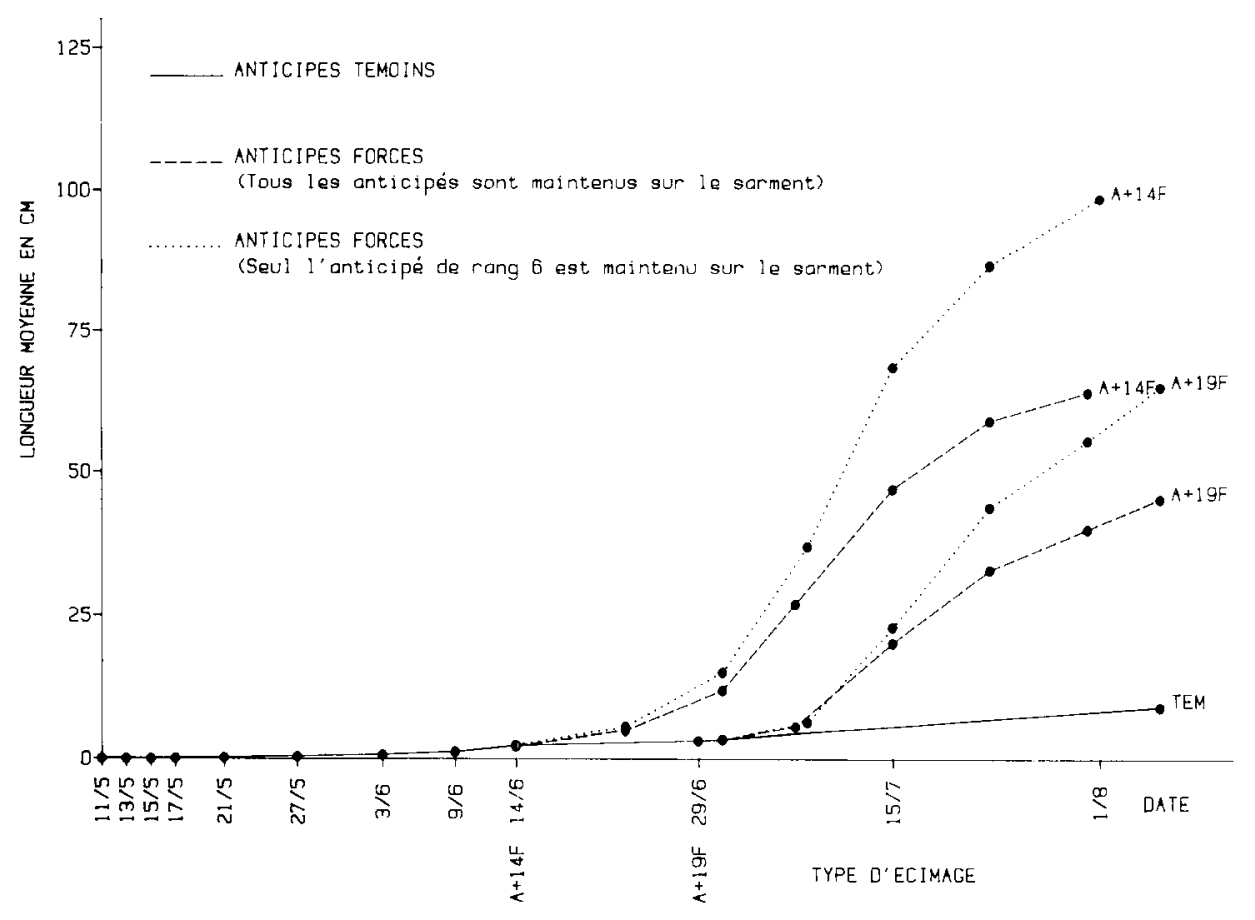

Figure 3

Cinétique de croissance des rameaux anticipés de rang 6 suite aux écimages " apex +14 et 19 feuilles 》, avec ou sans maintien de tous les anticipés sur le sarment.
Growth kinetics of the lateral shoots at position 6 after the decapitations " apex +14 or 19 leaves " with and without all lateral shoots kept on the main shoot. 
TABLEAU 1

Fertilité exprimée et fertilité pratique des rameaux anticipés de rang 6 obtenues lors des écimages « apex +14 et 19 feuilles », comparaison entre 2 lots expérimentaux.

Observed fertility and actual fertility of the lateral shoots at position 6 obtained for the decapitations « apex +14 or 19 leaves ", comparison between two experimental sets.

\begin{tabular}{|c|c|c|c|c|}
\hline & \multicolumn{2}{|c|}{ Ecimage 《apex +14 feuilles » } & \multicolumn{2}{|c|}{ Ecimage $«$ apex +19 feuilles $»$} \\
\hline & $\begin{array}{l}\text { Fertilité } \\
\text { exprimée }\end{array}$ & $\begin{array}{l}\text { Fertilité } \\
\text { pratique }\end{array}$ & $\begin{array}{l}\text { Fertilité } \\
\text { exprimée }\end{array}$ & $\begin{array}{l}\text { Fertilité } \\
\text { pratique }\end{array}$ \\
\hline $\begin{array}{l}\text { Tous les anticipés sont maintenus } \\
\text { sur le sarment }\end{array}$ & 1,6 & 1 & 1,6 & 0,3 \\
\hline $\begin{array}{l}\text { Seul l'anticipé situé sous la décapi- } \\
\text { tation est maintenu sur le sarment }\end{array}$ & 1,5 & 1,1 & 1,3 & 0,2 \\
\hline
\end{tabular}

général. En ce qui concerne la chute des grappes, il est important de mentionner qu'elle a lieu très tôt ; nous pouvons observer directement le commencement du phénomène en repérant les grappes très petites $(2 \dot{z}$ $5 \mathrm{~mm}$ de longueur) dont le développement est arrêté. Les grappes destinées à tomber peuvent être pratiquement toutes décelées dès le 7 juillet pour l'écimage « apex $+14 \mathrm{~F}$ » réalisé le 14 juin et dès le 15 juillet pour l'écimage « apex $+19 \mathrm{~F}$ » pratiqué le 29 juin. Le processus de chute des grappes est donc largement amorcé dès le début de la période de croissance active.

En définitive, il apparaît peu probable que la baisse de vitesse de croissance observée, surtout pour l'écimage "apex + $19 \mathrm{~F}$ ", soit la cause principale de la chute des grappes puisque l'augmentation de la vitesse de croissance n'a pas enrayé cette chute. Etant donné la précocité du phénomène, on peut en rechercher les causes dans les influences subies par les anticipés avant et tout au début des écimages. En effet, pour les écimages tardifs, les anticipés sont maintenus en croissance ralentie durant une période de plus en plus longue et les expériences conduites en ne gardant qu'un seul anticipé sur le sarment écimé n'ont pas modifié le début de la croissance. Il est donc possible que l'accélération de la croissance due à l'écimage intervienne trop tard lorsque le phénomène est déjà amorcé.

\section{Discussion}

Fertilité potentielle des anticipés avant écimage (fig. 1), fertilité exprimée et fertilité pratique des anticipés développés après écimage (OLIVAIN \& BESSIS, 1988) sont représentées sur un même graphique (fig. 4). La comparaison de ces 3 courbes avec la cinétique de croissance des anticipés développés après écimage (fig. 2) nous autorise les interprétations suivantes :

- Les écimages des $11,13,15$ et 17 mai interviennent sur des anticipés dont la fertilité potentielle est égale à 0 , pourtant, après écimage, la fertilité est de 0,5 à 0,8 grappe par anticipé, le nombre de baies par grappe n'étant pas supérieur à 10 (OLIVAIN \& BESSIS, 1988). Jusqu'à la fin mai, la croissance des anticipés

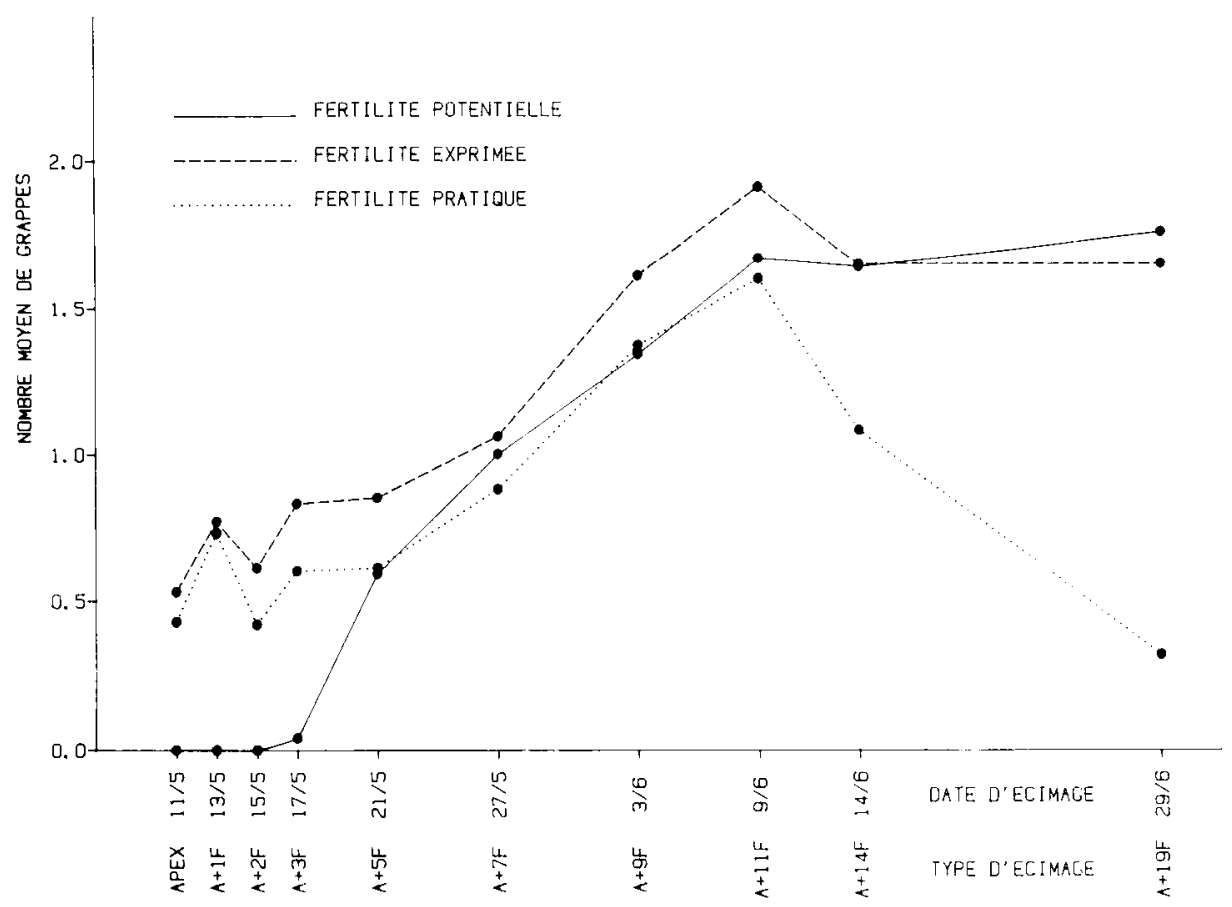

Figure 4

Fertilité potentielle, fertilité exprimée et fertilité pratique des anticipés de rang 6. 
forcés est proche de celle des anticipés témoins : on peut donc penser que du point de vue fertilité la mise en place et la différenciation des inflorescences n'est pas très différente. Fin mai, l'accélération de la croissance intervient donc sur des anticipés avec une fertilité potentielle de l'ordre de 0,5 à 0,9 grappe par anticipé, les inflorescences étant au stade « initiation des premières ramifications secondaires ». La fertilité obtenue après écimage serait alors l'expression exacte de la fertilité potentielle au moment de l'entrée en croissance. Il faut donc admettre que l'accélération de la croissance favorise d'une part, la transformation des " anlage " en vrille et d'autre part, limite la ramification des inflorescences, les différents méristèmes présents évoluant rapidement en boutons floraux.

- Les écimages des 21, 27 mai, 3 et 9 juin s'adressent à des anticipés qui ont un nombre croissant de grappes potentielles de plus en plus organisées. La fertilité obtenue après écimage augmente progressivement pour atteindre une valeur maximale au 9 juin, nous avons également noté que la grosseur des grappes est de plus en plus importante. Pour tous ces écimages, les anticipés forcés entrent assez rapidement en croissance, ce qui explique que la fertilité obtenue soit l'expression exacte de la fertilité potentielle au moment de l'écimage ou quelques jours plus tard. En ce qui concerne la grosseur des grappes, elle est dépendante de l'état d'organisation des inflorescences mais l'accélération de la croissance a également limité leur ramification puisque nous n'obtenons les valeurs maximales qu'au 9 juin lorsqu'il existe un maximum d'inflorescences au stade "ramifications tertiaires " (OLIVAIN \& BESSIS, 1987). Sur les anticipés témoins, les boutons floraux se différencient après le 9 juin lorsque ceux-ci manifestent une certaine croissance : l'écimage au 9 juin permet une croissance continue des anticipés et correspond à l'expression maximale de la fertilité du point de vue nombre et grosseur des grappes.

- Les écimages des 14 et 19 juin sont effectués sur des anticipés qui ont un maximum de grappes potentielles: au niveau du premier organe oppositifolié, environ $50 \%$ d'entre elles possèdent des boutons floraux. Ces grappes ne se maintiennent pas sur les anticipés développés après écimage. Pour ces 2 cas, les anticipés entrent en croissance dans un laps de temps assez court mais la vitesse maximale atteinte est plus faible, surtout pour l'écimage « apex $+19 \mathrm{~F}$ » du 29 juin où la chute des grappes est pratiquement totale. Dans un premier temps nous avons pensé que cette croissance plus faible était la cause de la chute des grappes. Les expériences menées en ne gardant qu'un seul anticipé sur le sarment écimé ne semblent pas confirmer cette hypothèse. Il faut donc admettre que vers la mi-juin les grappes ne sont plus aptes à se développer ; cette perte d'aptitude pourrait être occasionnée par la présence d'une période de croissance ralentie plus ou moins longue située avant la réponse à l'écimage et durant cette période nous avons signalé que la différenciation des inflorescences est arrêtée, tout au moins au niveau du premier organe oppositifolié.

\section{CONCLUSION}

L'étude réalisée nous a permis de comprendre la fertilité des rameaux anticipés. Sur sarments non écimés, les anticipés de rang 6 atteignent leur fertilité potentielle maximale début juin. Pour que cette fertilité s'exprime il faut provoquer le développement des anticipés par des écimages. Les résultats obtenus peuvent être interprétés de la façon suivante :

- La fertilité exprimée est voisine de la fertilité potentielle présente au moment de l'entrée en croissance des anticipés et cette époque ne coüncide pas obligatoirement avec la date d'écimage. Par exemple, pour les écimages précoces du début mai, les anticipés entrent en croissance lentement, ce qui permet la mise en place d'un certain nombre d'inflorescences.

- La fertilité exprimée maximale n'est obtenue que lorsqu'elle est atteinte au niveau des anticipés témoins, début juin. Les écimages précoces induisent une perte de fertilité expliquée par le fait que la croissance des anticipés favorise la transformation des « anlage » en vrille et limite la ramification des inflorescences au profit de la mise à fleurs.

- La fertilité pratique maximale ne peut être maintenue que pendant une courte période. Aux écimages tardifs, il existe une perte de fertilité provoquée par la chute des grappes lors de l'entrée en croissance des anticipés. La chute des grappes serait en relation avec un arrêt de leur développement, cet arrêt se produisant durant la période de croissance ralentie des anticipés sur sarments non écimés.

Ces observations tendent à montrer que la formation et l'organisation des inflorescences sont favorisées par une vitesse de croissance lente mais que le plein développement de ces inflorescences est conditionné par une croissance continue à vitesse plus élevée. Ces résultats peuvent être rapprochés de ceux obtenus par POUGET (1981) sur les bourgeons latents pincipaux qui montre qu'un débourrement lent à basse température produit un nombre de fleurs nettement plus grand qu'un débourrement rapide à température élevée.

Reçu le 9 octobre 1986 Accepté le 3 novembre 1987

\section{RÉFÉRENCES BIBLIOGRAPHIQUES}

Alleweldt G., Balkema G. H., 1965. Über die Anlage von Infloreszenz- und Blütenprimordien in der Rebe. Z. Acker- $u$. Pflanzenbau, 123, 59-74.

Bouard J., 1966. Recherches physiologiques sur la vigne et en particulier sur l'aoûtement des sarments. Thèse, Bordeaux, $310 \mathrm{p}$.

Fournioux J. C., Bessis R., 1980. Effet d'un effeuillage régulier sur la croissance et l'organogenèse du rameau de la vigne. Bull. Soc. bot. Fr., 127, Lettres bot., 1, 23-34.

Olivain C., Bessis R., 1987. L'organogenèse inflorescentielle dans les bourgeons anticipés de vigne (Vitis vinifera $\mathrm{L}$. cépage Pinot). Vitis, 26, $98-106$

Olivain C., Bessis R., 1988. Fertilité des rameaux anticipés de vigne (Vitis vinifera L.) : 1) expression au vignoble. Agronomie, 8 (2), 133138

Pouget R., 1981. Action de la température sur la différenciation des inflorescences et des fleurs durant les phases de pré-débourrement et de post-débourrement des bourgeons latents de la vigne. Connaissance Vigne et Vin, 15, 2, 65-79. 